\title{
Cystic Fibrosis in Female Gender
}

\section{Tiziana Ciarambino ${ }^{1}$, Ombretta Para $^{2}$ and Mauro Giordano MD PhD ${ }^{3 *}$}

${ }^{1}$ Dirigente Medico, Ospedale Clinicizzato Marcianise, ASL Caserta, Italy

${ }^{2}$ Dirigente Medico, Ospedale Careggi, University of Firenze, Italy

${ }^{3}$ Department of Advanced Medical and Surgical Sciences, University of Campania

"L. Vanvitelli", Naples, Italy

*Corresponding Author: Mauro Giordano MD PhD, Department of Advanced

Medical and Surgical Sciences, University of Campania "L. Vanvitelli“, Naples, Italy.

E-mail: mauro.giordano@unicampania.it
Received: July 20, 2021

Published: September 08, 2021

(C) All rights are reserved by Mauro

Giordano., et al.

\begin{abstract}
Introduction: Cystic fibrosis (CF) is a lethal autosomal recessive disease. It has been reported that females are diagnosed later than males (2) and have a significantly decreased survival.

Methods: Clinical trials were identified by PubMed until June 30 2020. The search keywords were "sex, gender differences, cystic fibrosis".

Result: It has been suggested, that cystic fibrosis is diagnosed later in female children, because underdiagnosed. Unfortunately, cystic fibrosis determines airway inflammation and chronic respiratory infection, such as Pseudomonas aeruginosa. Different factors are related to these inflammations. However, mortality and cystic fibrosis-related diabetes (CFRD) prevalence were higher, in female subjects than males.

Conclusion: Female subjects have significantly higher mortality than males, with about 4-year difference in median survival. However, adolescent girls with CF have a greater emotional impact compared to boy.
\end{abstract}

Keywords: Sex; Gender Differences; Cystic Fibrosis

\section{Introduction}

Cystic fibrosis (CF) is a lethal autosomal recessive disease, affecting 1 in 3.200 births [1]. Gender differences are a crucial factor on the diagnosis and progression of CF. In fact, it has been reported that cystic fibrosis is diagnosed later in female children than males [2]. However, female with age between 1 to 20 years [3] have a significantly decreased survival. To this regard, it has been described that female subjects are less adherent to therapy [4] and they have a life span shorter (about 3 to 5 years) than that of males [3]. We report a minireview on the cystic fibrosis in female subjects.

\section{Methods}

Clinical trials were identified by PubMed until June 302020. The search keywords were "sex, gender differences, cystic fibrosis". The studies were selected and their references were reviewed for potential inclusion. Studies written in languages other than English were excluded. Two authors (O.P. and T.C.) reviewed all study abstracts. Studies were included if analyzed and reported gender differences in cystic fibrosis. All selected studies are qualitatively analyzed. 


\section{Results and Discussion}

\section{Cause of the gender gap in cystic fibrosis}

The underlying cause of the gender gap in cystic fibrosis can be reported [5]:

- Genetic modifications

- Socio-environmental effects of gender

It has been reported that cystic fibrosis is diagnosed later in female children than males probably for reduced recognition of respiratory symptoms in female infants. Unfortunately, the prevalence of the adult CF diagnosis is significantly greater in females [6]. Moreover, the rate of $\mathrm{FEV}_{1}$ is similar by gender, but female subjects have a survival advantage of about 9-13 years [7]. In fact, cystic fibrosis induces an airway inflammation and chronic respiratory infection, such as Pseudomonas aeruginosa. To this regard, it has been reported that, females with CF, acquired chronic Pseudomonas aeruginosa infection at an earlier age. In particular, female subjects express a quickly decline in lung function after P. aeruginosa colonization [8].

\section{Estrogen and cystic fibrosis}

Sex hormones induce alterations on the components of the muco-ciliary apparatus, in airway epithelial cell apical sodium and chloride transport [9], alter airway surface liquid (ASL) volume [10] and change cilia beat frequency in the airways [11]. The role of estrogen in female subjects in cystic fibrosis is reported in table 1. These factors can determine, in females with cystic fibrosis, a major disadvantage than males. In fact, these characteristics can induce an increase on the mucus viscosity [12]. To this regard, it has been reported that estrogens determine conversion of P. aeruginosa, from a non-mucoid to mucoid form. Unfortunately, this form is the more drug resistant [13]. Moreover, female subjects acquire infection by $P$. aeruginosa, $H$. influenzae, $A$. xylosoxidans, $B$. cepacian, Aspergillus species and methicillin resistant Staphylococcus aureus (MRSA), at an earlier age [14]. Another role of estrogen in cystic fibrosis was related to immune system. In particular, it has been reported, that estrogen induce inhibition of interleukin-8 production in CF bronchial epithelial cells in vitro and it would inhibit neutrophil recruitment and inflammatory responses in female subjects [15].

\begin{tabular}{|l|c|}
\hline Air surface liquid & Decrease \\
\hline Mucus viscosity & Enhanced \\
\hline $\begin{array}{l}\text { Conversion of } P \text {. aeruginosa } \\
\text { from a nonmucoid to mucoid } \\
\text { form }\end{array}$ & Increased \\
\hline $\begin{array}{l}\text { Drug resistant and pathogenic } \\
\text { form }\end{array}$ & Increased \\
\hline $\begin{array}{l}\text { P. aeruginosa and other } \\
\text { infection }\end{array}$ & $\begin{array}{l}\text { An earlier } \\
\text { age }\end{array}$ \\
\hline Interleukin-8 production & Inhibited \\
\hline
\end{tabular}

Table 1: Estrogen role.

\section{Other factors and gender}

It has been reported that there was also a sex difference in adults' diabetes with severe CF. In particular, mortality and prevalence on the cystic fibrosis were higher, in female diabetic than males. To this regard, it has been observed that insulin clearance increases with age. This increment was described especially in females, and it contribute to the deterioration in glucose tolerance [16].

However, disease severity has an impact on the quality of life (HRQoL). To this regard, females generally have poorer HRQoL. In fact, female subjects have a more accurate perception of clinical health status [17]. This perception has a greater emotional impact on adolescent girls compared to boys.

\section{Conclusion}

Females with cystic fibrosis have significantly higher mortality than males. In fact, it has been reported about four-year difference in median survival by gender. However, cystic fibrosis induces a major emotional impact on adolescent girls compared to boys. The adolescent girls are less adherent to therapy. Therefore, these gender differences may outline the reduced pulmonary function observed in girls with cystic fibrosis [18]. Future studies are need to evaluate the different outcome by gender in CF patients.

\section{Conflict of Interest}

The authors declare that haven't conflict of interest.

\section{Acknowledgement}

We thanks the doctor Cecilia Politi, MD Ospedale F. Veneziale Isernia, ASREM, Italy. 


\section{Bibliography}

1. Sontag MK., et al. "Two- tiered immunoreactive trypsinogenbased newborn screening for cystic fibrosis in Colorado: screening efficacy and diagnostic out- comes". Journal of Pediatrics 147 (2005): S83-S88.

2. Lai HC., et al. "Delayed diagnosis of US females with cystic fibrosis". American Journal of Epidemiology 156 (2002): 165173.

3. Rosenfeld M., et al. "Gender gap in cystic fibrosis mortality". American Journal of Epidemiology 145 (1997): 794-803.

4. Patterson JM., et al. "Associations of psychosocial factors with health outcomes among youth with cystic fibrosis". Pediatric Pulmonology 44 (2009): 46-53.

5. Wolfenden LL and Schechter MS. "Genetic and non-genetic determinants of outcomes in cystic fibrosis". Paediatric Respiratory Reviews 10 (2009): 32-36.

6. Jerry A Nick., et al. "Effects of Gender and Age at Diagnosis on Disease Progression in Long-term Survivors of Cystic Fibrosis". American Journal of Respiratory and Critical Care Medicine 182 (2010): 614-626.

7. Arias E., et al. "US decennial life tables for 1999-2001, United States life tables". National Vital Statistics Reports 57 (2008): $1-36$.

8. Demko CA., et al. "Gender differences in cystic fibrosis: Pseudomonas aeruginosa infection". Journal of Clinical Epidemiology 48 (1995): 1041-1049.

9. Sweezey NB., et al. "Sex hormones regulate CFTR in developing fetal rat lung epithelial cells". American Journal of Physiology 272 (1997): L844-851.

10. Coakley RD., et al. "17beta-Estradiol inhibits Ca2 + -dependent homeostasis of airway surface liquid volume in human cystic fibrosis airway epithelia". Journal of Clinical Investigation 118 (2008): 4025-4035.

11. Jain R., et al. "Sex hormone- dependent regulation of cilia beat frequency in airway epithelium". American Journal of Respiratory Cell and Molecular Biology 46 (2012): 446-453.
12. Nick JA., et al. "Effects of gender and age at diagnosis on disease progression in long-term survivors of cystic fibrosis". American Journal of Respiratory and Critical Care Medicine 182 (2010): 614-626.

13. Dasenbrook EC., et al. "Association between respiratory tract methicillin-resistant Staphylococcus aureus and survival in cystic fibrosis". JAMA 303 (2010): 2386-2392.

14. Maselli JH., et al. "Risk factors for initial acquisition of Pseudomonas aeruginosa in children with cystic fibrosis identified by newborn screening". Pediatric Pulmonology 35 (2003): 257262.

15. Wang Y., et al. "Estrogen aggra- vates inflammation in Pseudomonas aeruginosa pneumonia in cystic fibrosis mice". Respiratory Research 11 (2010): 166.

16. Alberto Battezzati., et al. "Age- And Sex-Dependent Distribution of OGTT-Related Variables in a Population of Cystic Fibrosis Patients". The Journal of Clinical Endocrinology and Metabolism 100.8 (2015): 2963-2971.

17. L Gee., et al. "Quality of Life in Cystic Fibrosis: The Impact of Gender, General Health Perceptions and Disease Severity". Journal of Cystic Fibrosis 2.4 (2003): 206-213.

18. Joän M Patterson., et al. "Gender Differences in Treatment Adherence Among Youth With Cystic Fibrosis: Development of a New Questionnaire". Journal of Cystic Fibrosis 7.2 (2008): 154164.

\section{Volume 5 Issue 10 October 2021}

(C) All rights are reserved by Mauro Giordano., et al. 